\title{
Space-to-Ground Quantum Key Distribution
}

Austrian - Chinese collaboration Project

Thomas Scheidl

IQOQI - Institute for Quantum Optics and Quantum Information Austrian Academy of Sciences

Vienna, Austria 


\section{CAS - AAS Collaboration}
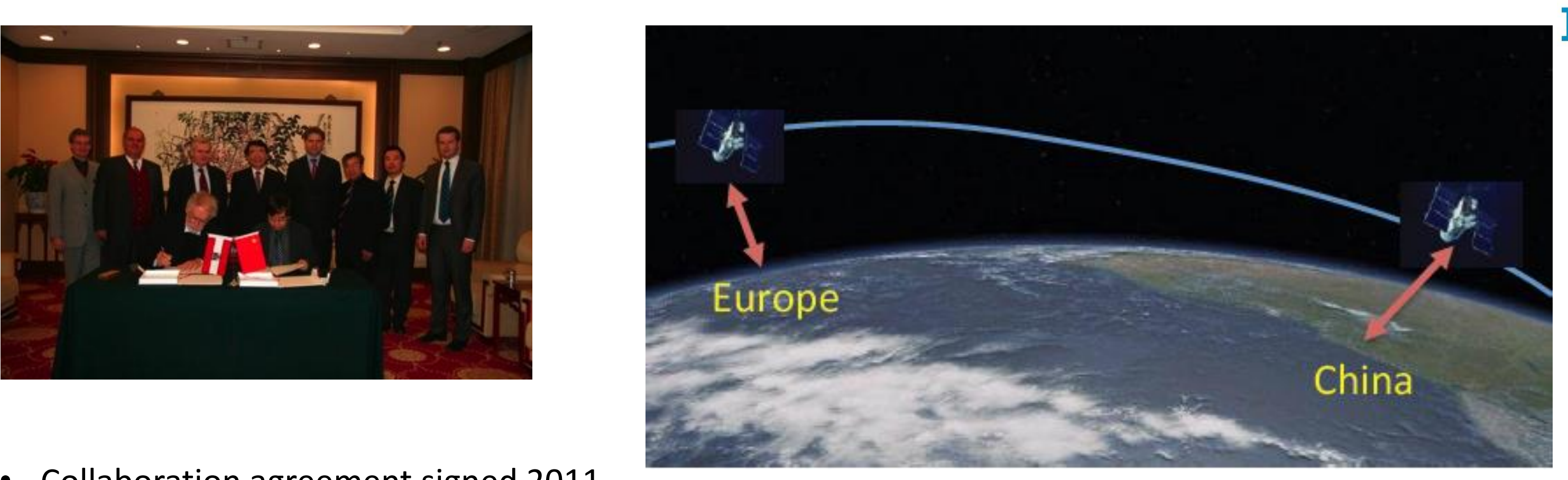

- Collaboration agreement signed 2011

- Satellite is developed and built in China

- Goal: Inter-continental quantum key exchange

- Austria provide Optical Ground Stations in Europe

- Bundesministerium Bildung, Wissenschaft und Forschung 


\section{Key Relay Protocol}

Individual keys are generated with the BB84 Decoy-state Protocol
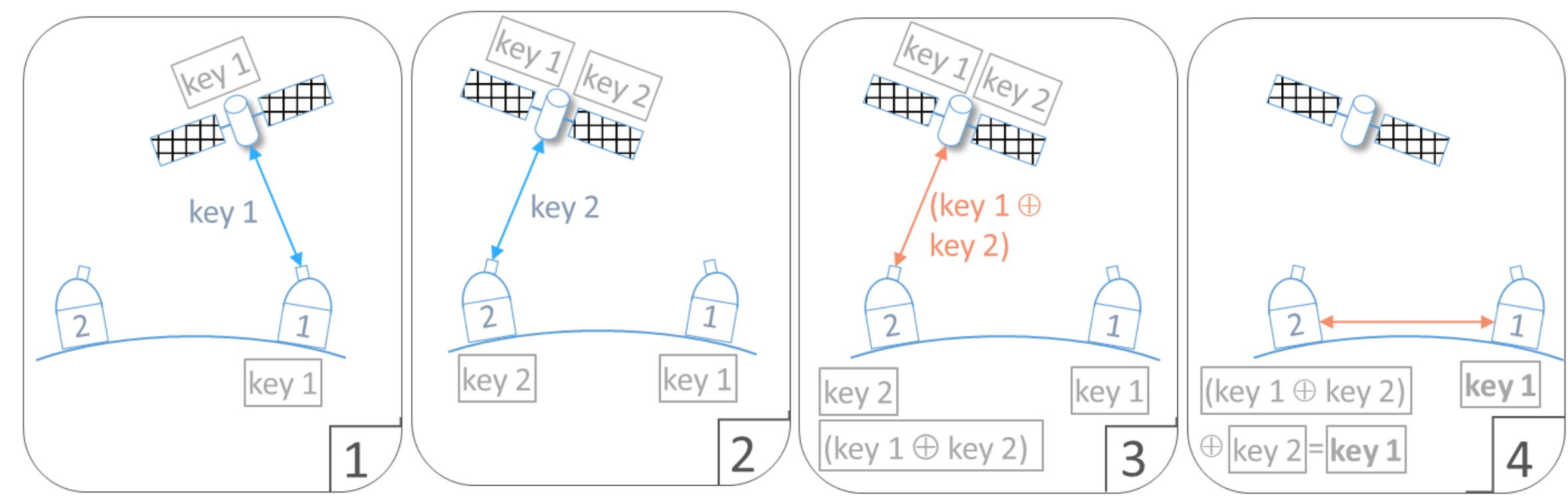


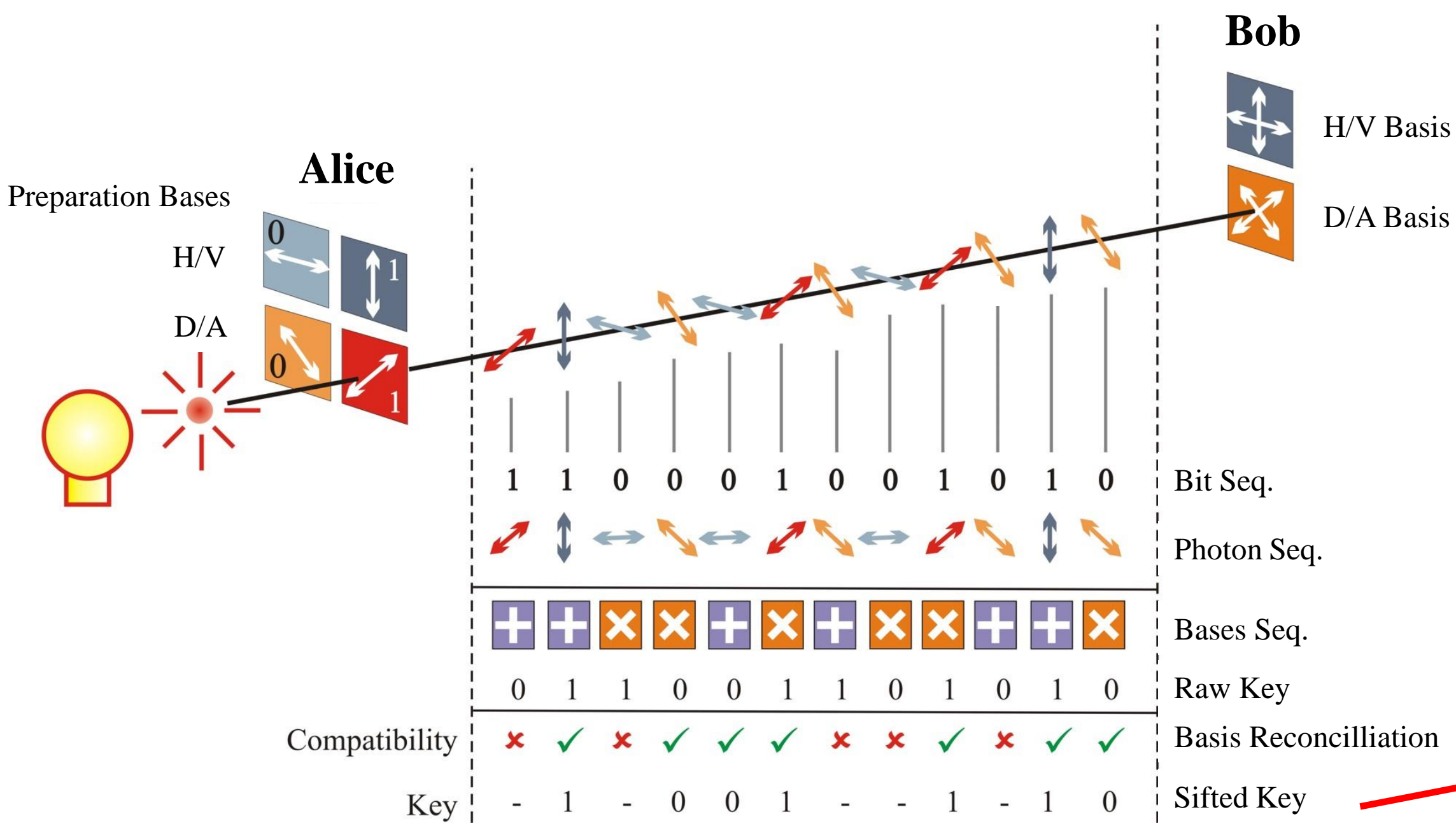

- Error correction

Key $\begin{array}{llllllllllllll:l} & - & 1 & - & 0 & 0 & 1 & - & - & 1 & - & 1 & 0 & \text { Sifted Key }\end{array}$

- Privacy amplification 
- Realistic implementations based on weak laser pulses with $\mu<1$

- Introduce pulses with different mean photon numbers

e.g.: $\mu_{\text {signal }}=0.8, \mu_{\text {decoy }}=0.1, \mu_{\text {vacuum }}=0$

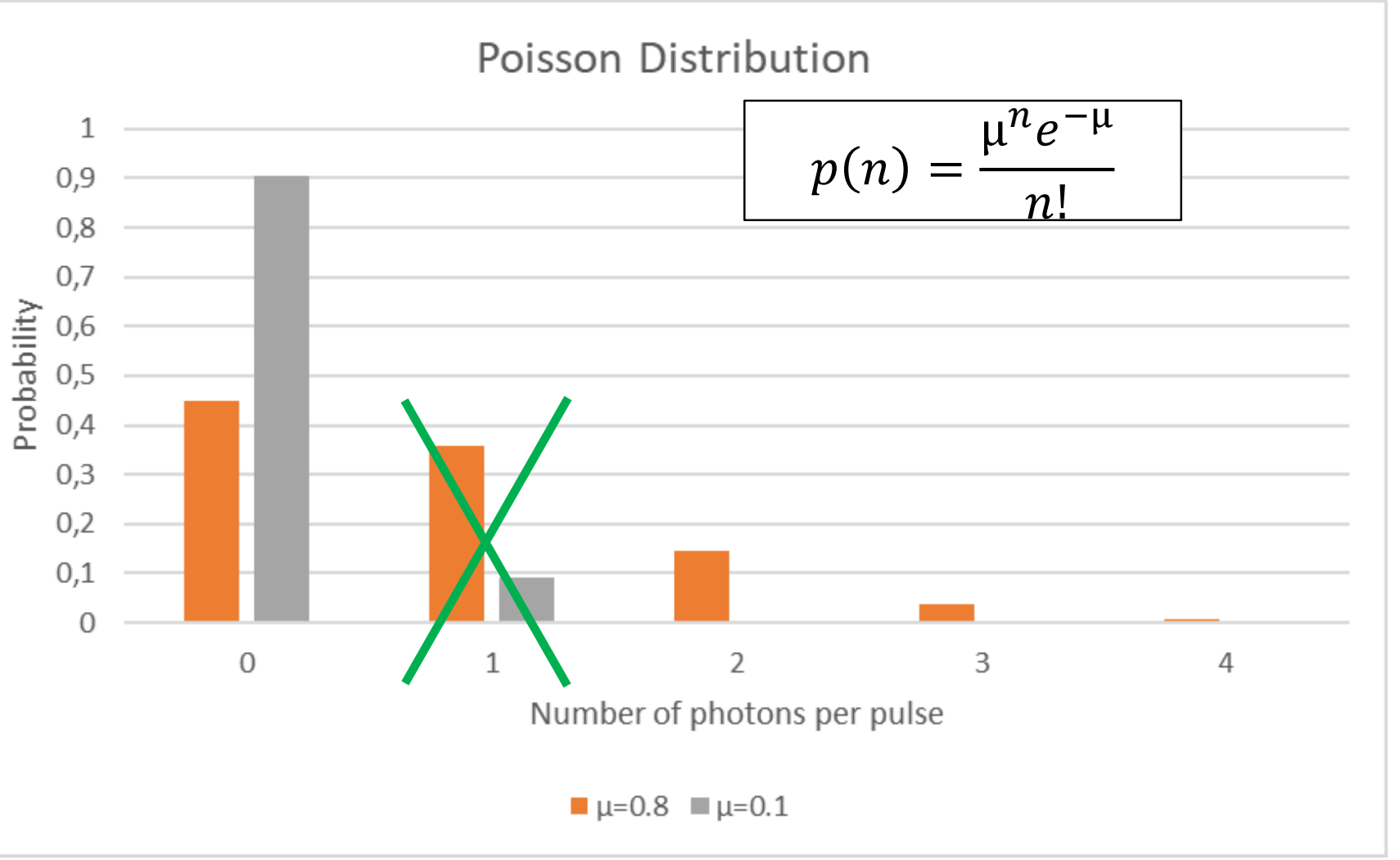

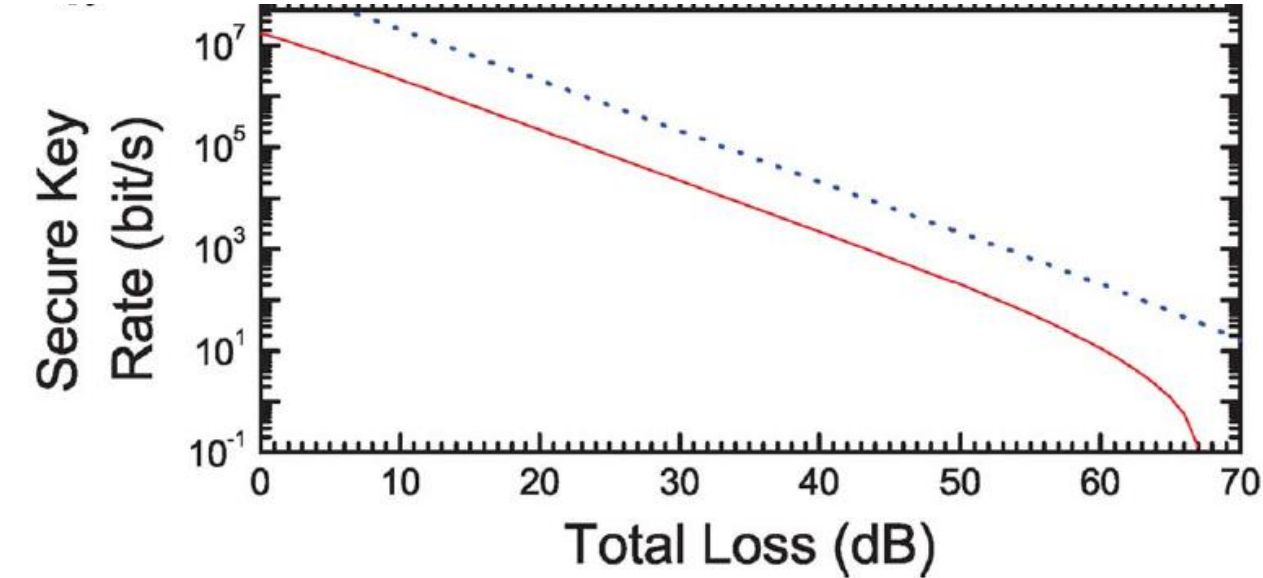

Journal of Lightwave Technology, Vol. 31, No. 9, May 1, 2013 


\section{Payload characteristics:}

- Quantum signal: $850 \mathrm{~nm}, 100 \mathrm{MHz}, 10 \mu \mathrm{rad}$

- Downlink beacon/synchronization laser: $532 \mathrm{~nm}, 10 \mathrm{kHz}, 3 \mathrm{mrad}$

- Uplink beacon: $671 \mathrm{~nm}, 3 \mathrm{~W}, 3 \mathrm{mrad}$

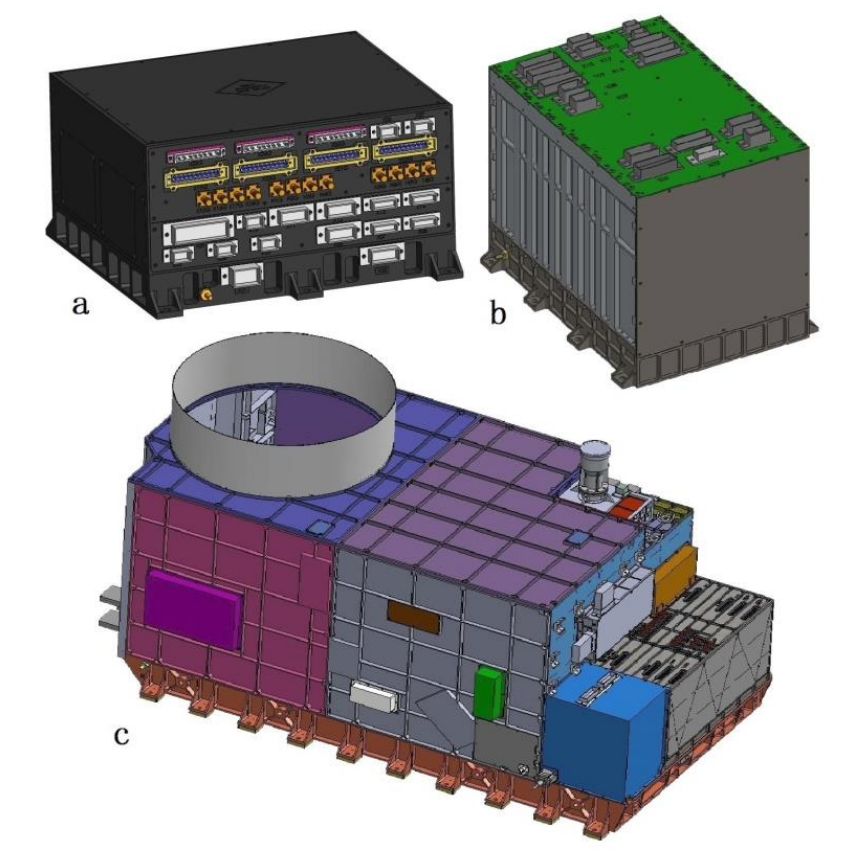

Figure: S.-K. Liao et al., "Satellite-Relayed Intercontinental Quantum Network," Phys. Rev. Lett. 120, 030501, 2018.

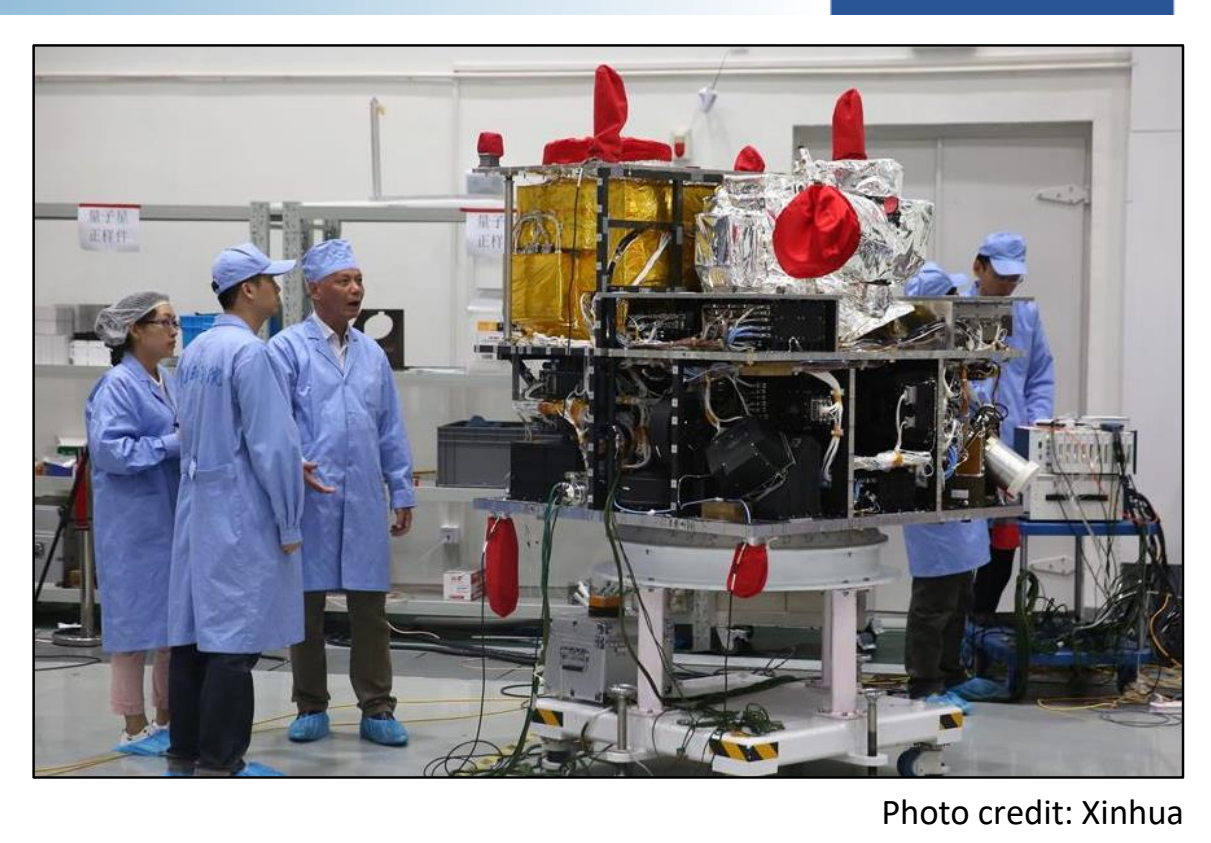

Orbit characteristics:

- Sun-synchronous

- $500 \mathrm{~km}$ height, $97.4^{\circ}$ inclination

- Decending node: 1:00h local time

- 500m prediction accuracy

- approx. 270 s link duration/pass

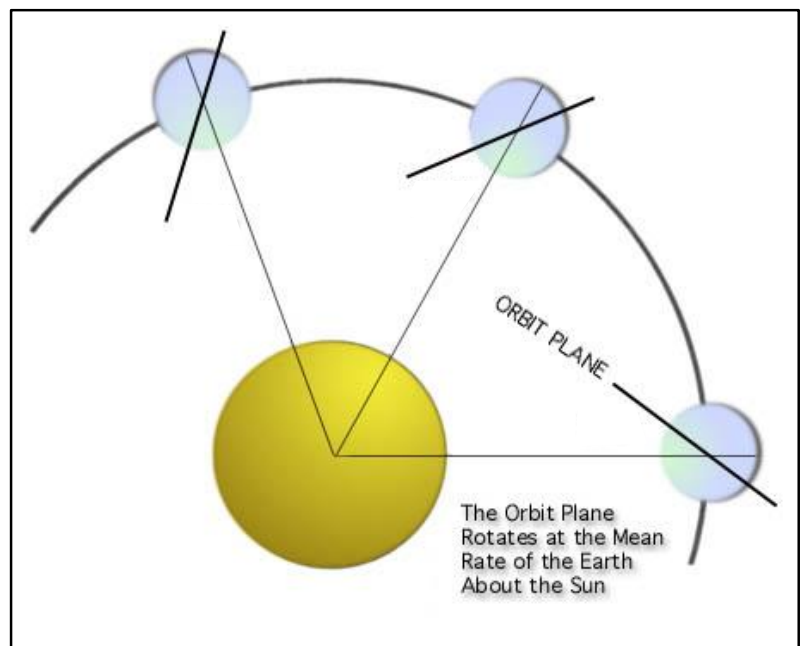




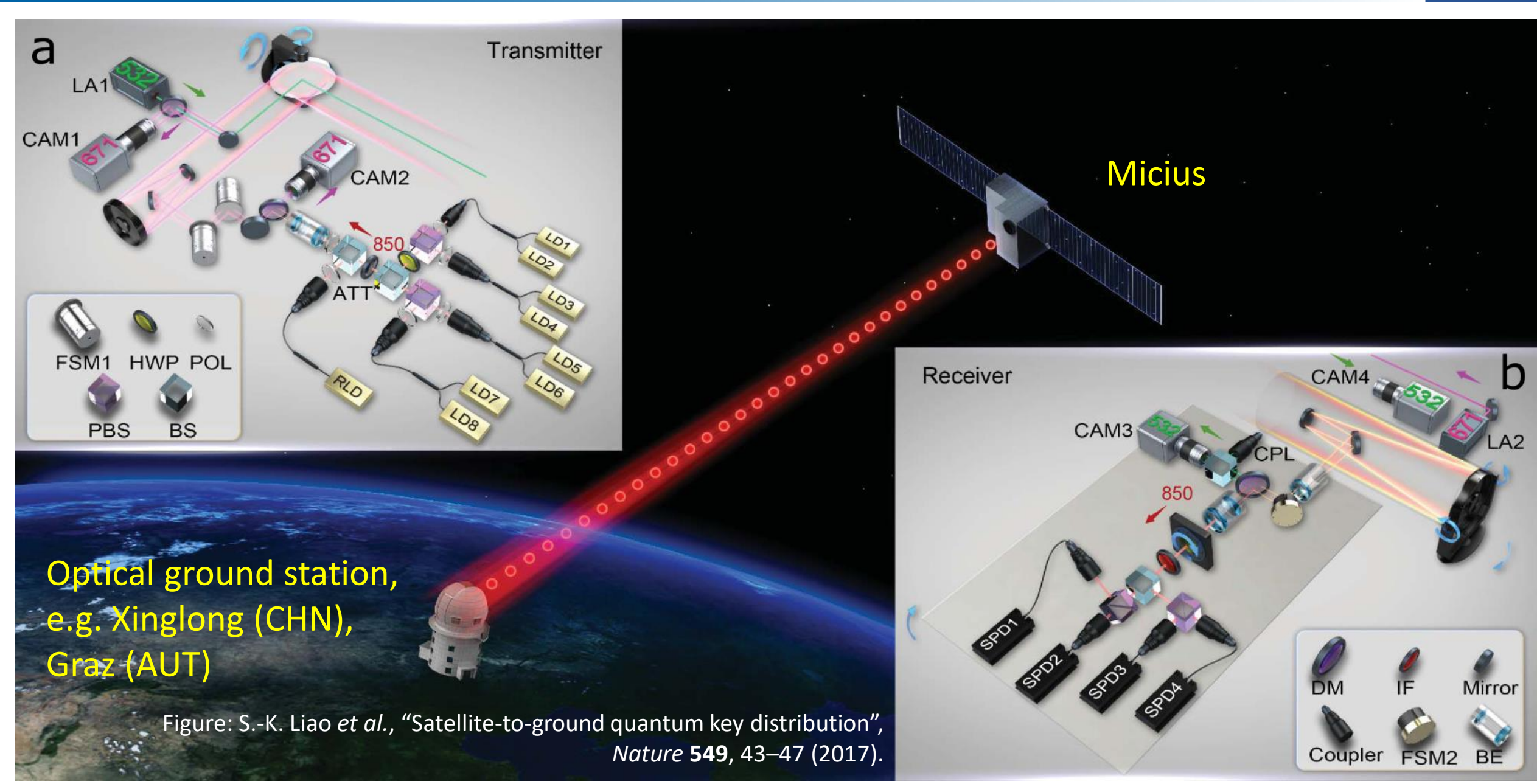

a: Transmitter optical setup showing the BB84 decoy source and the satellite tracking optics b: Receiver optics on ground, where the signal photons' polarization is being analyzed 


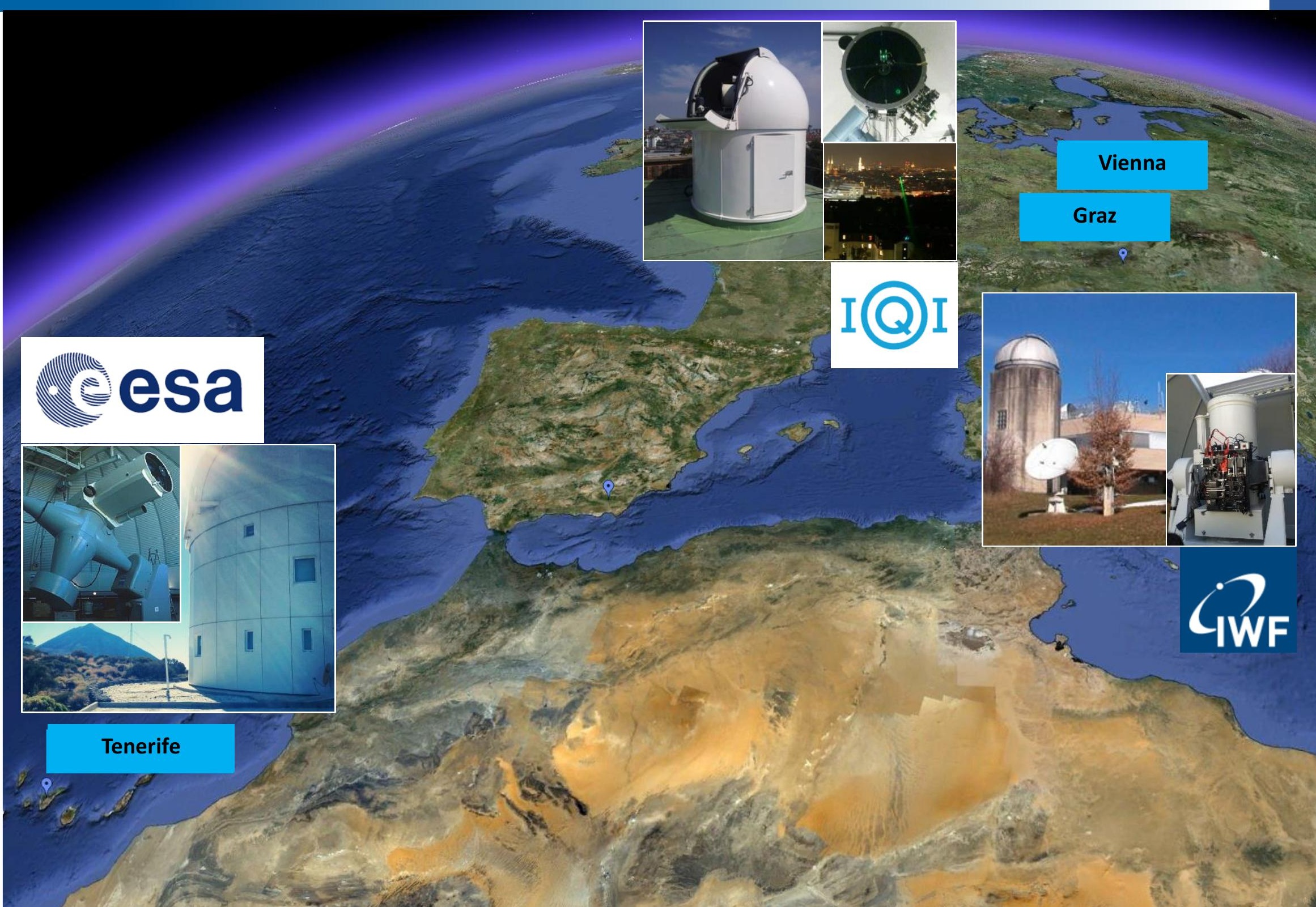




\section{Tracking Performance}

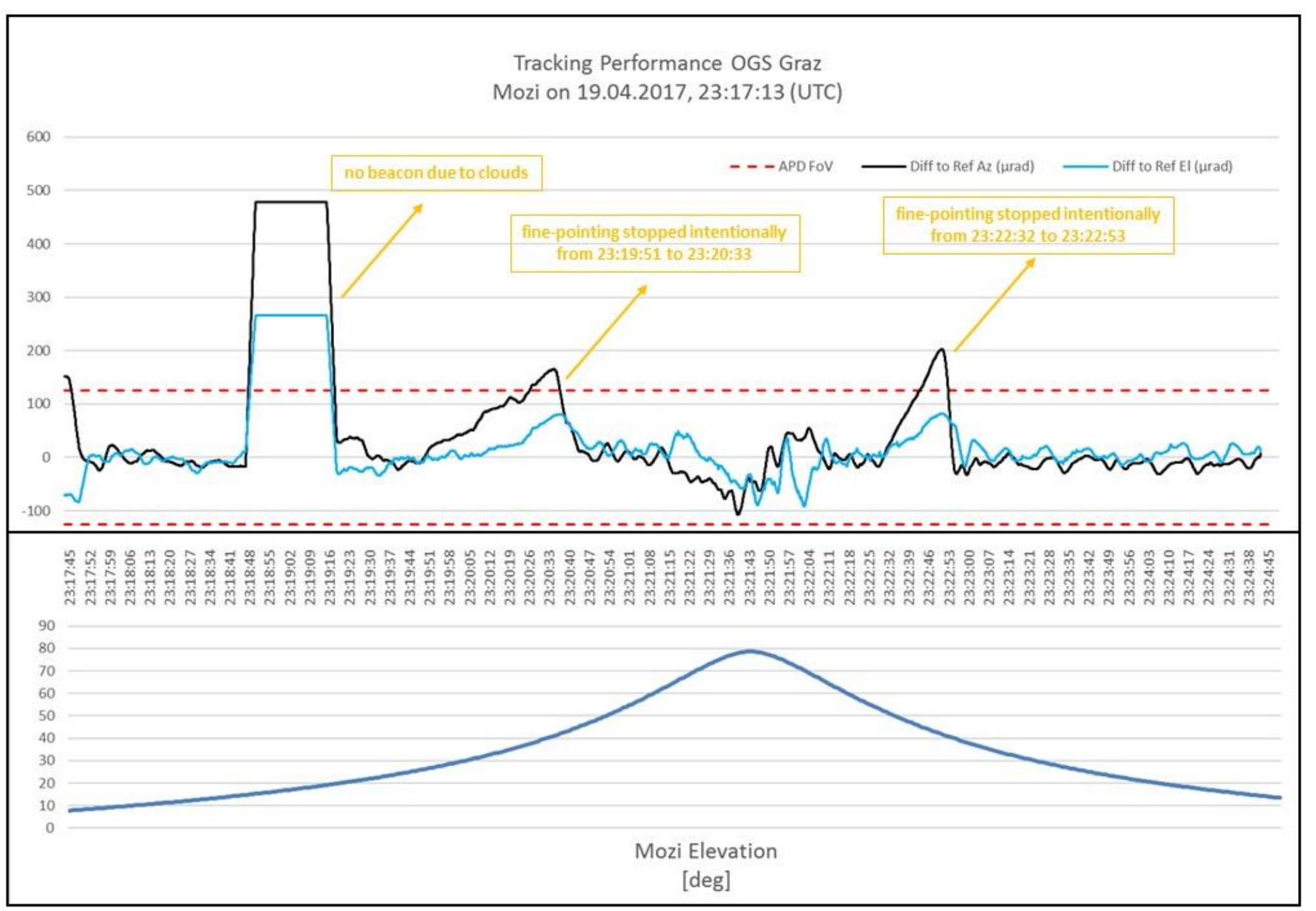




\section{Pol. Compensation Performance}

ÖSTERREICHISCHE AKADEMIE DER WISSENSCHAFTEN

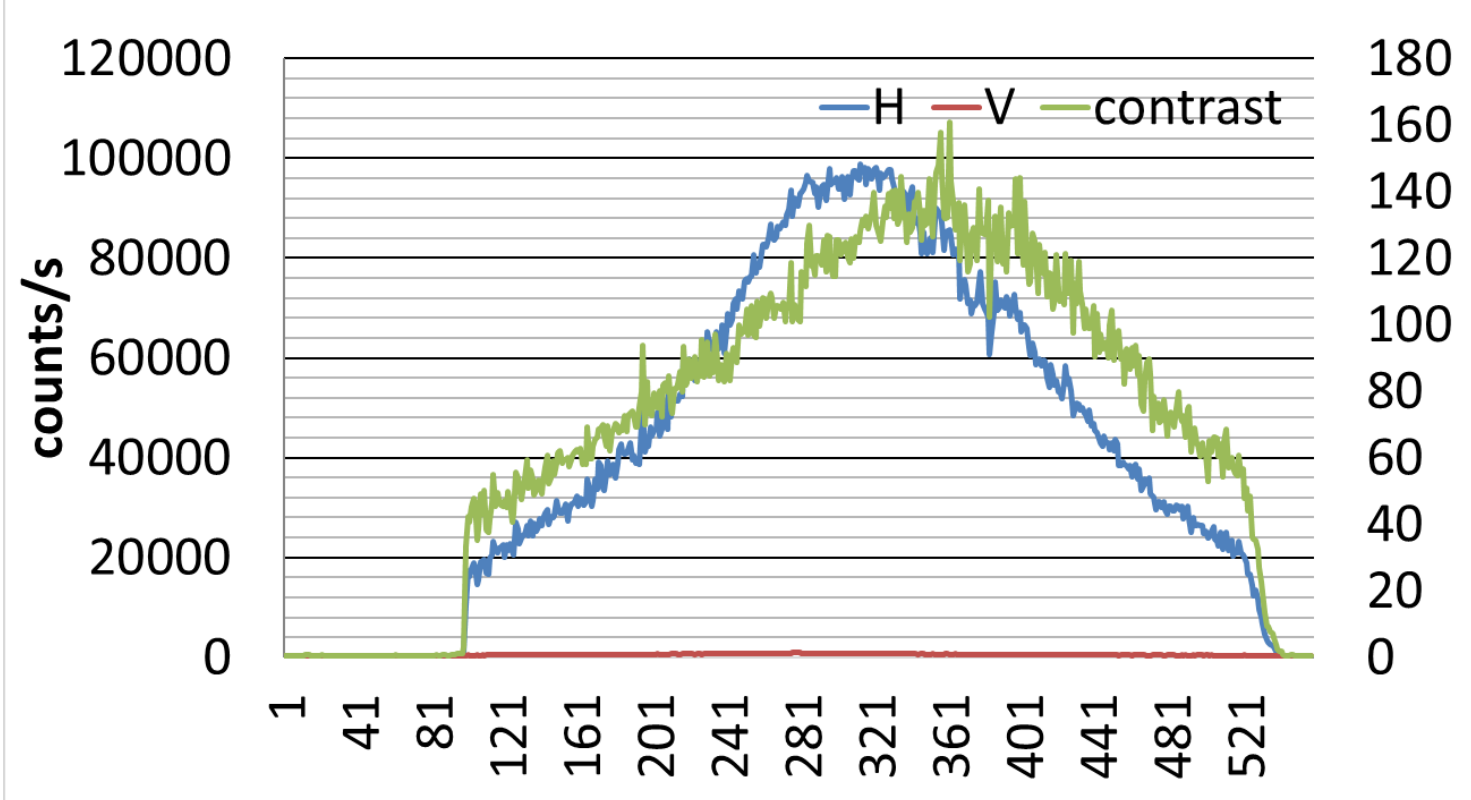

Half-Wave-Plate Angle

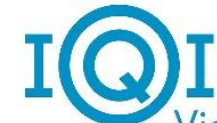

20

80

60

20

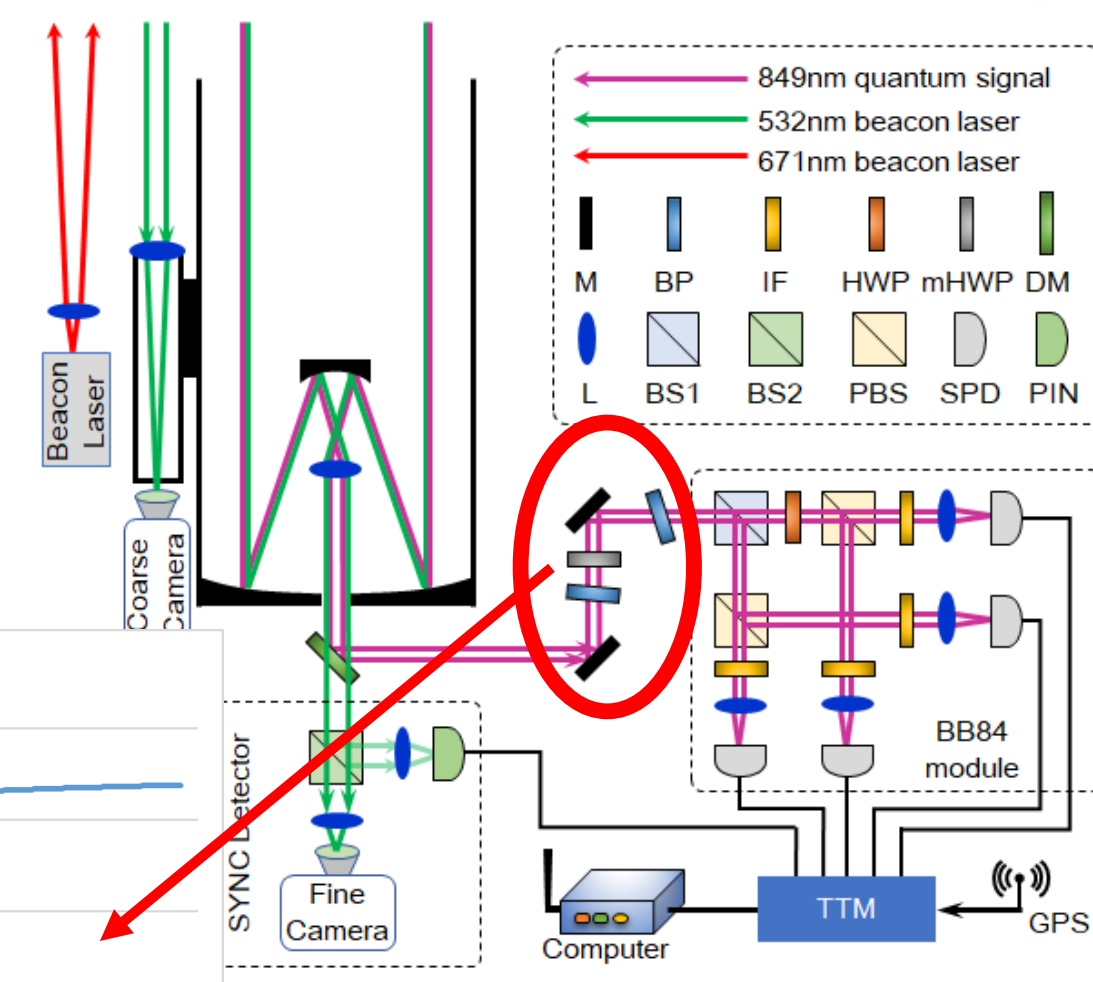




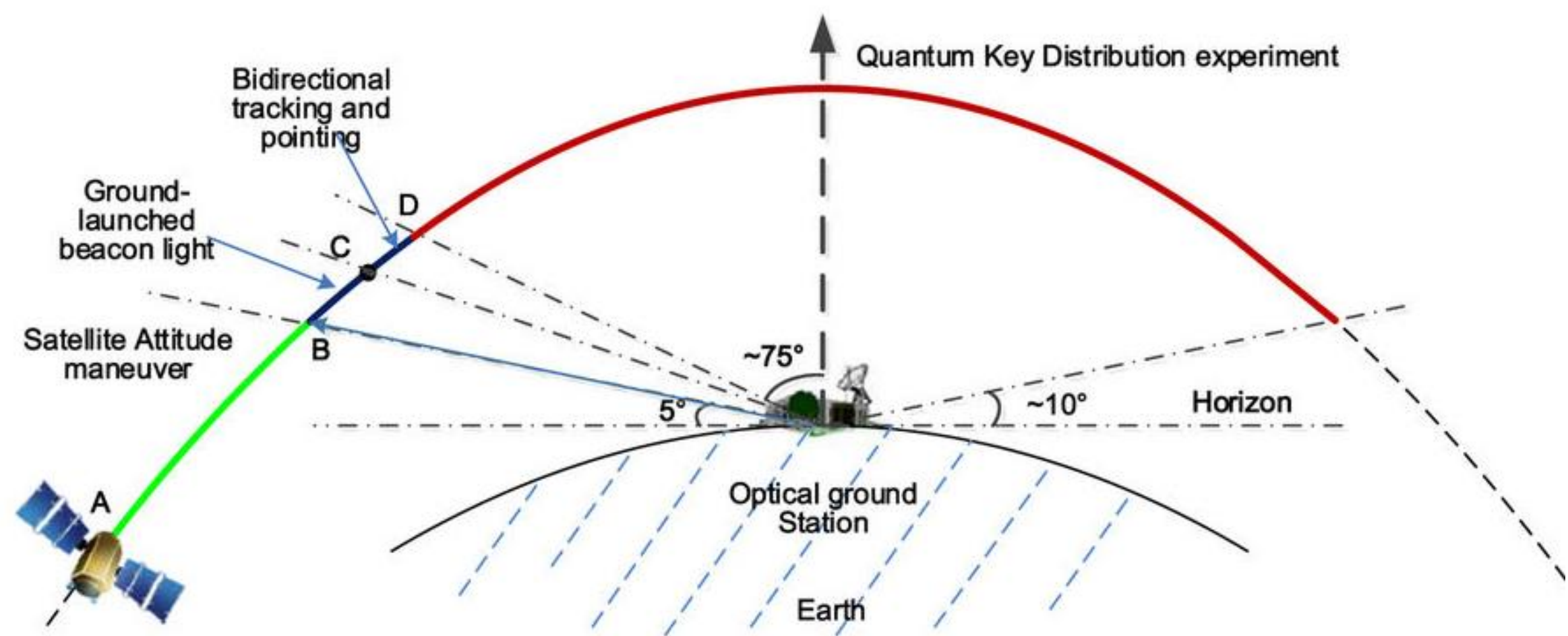




\section{Pictures \& Videos}

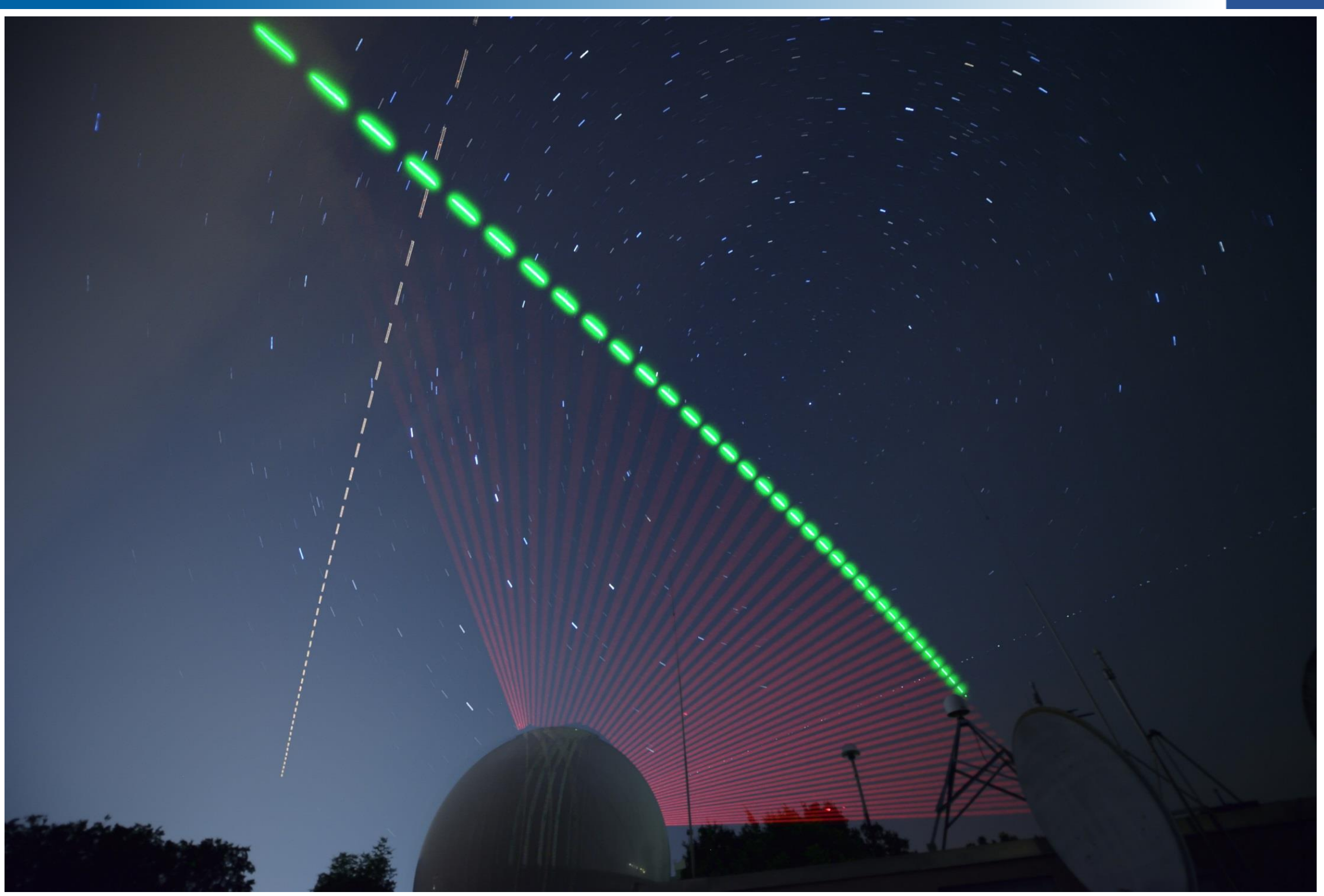




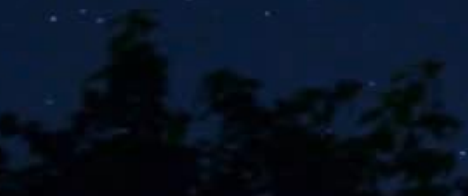

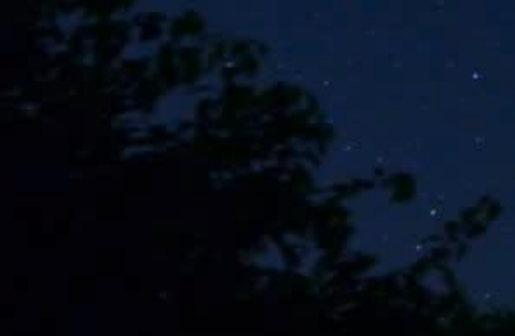




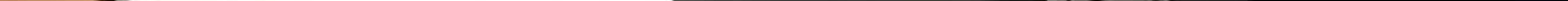




\section{Key Rates and QBER}
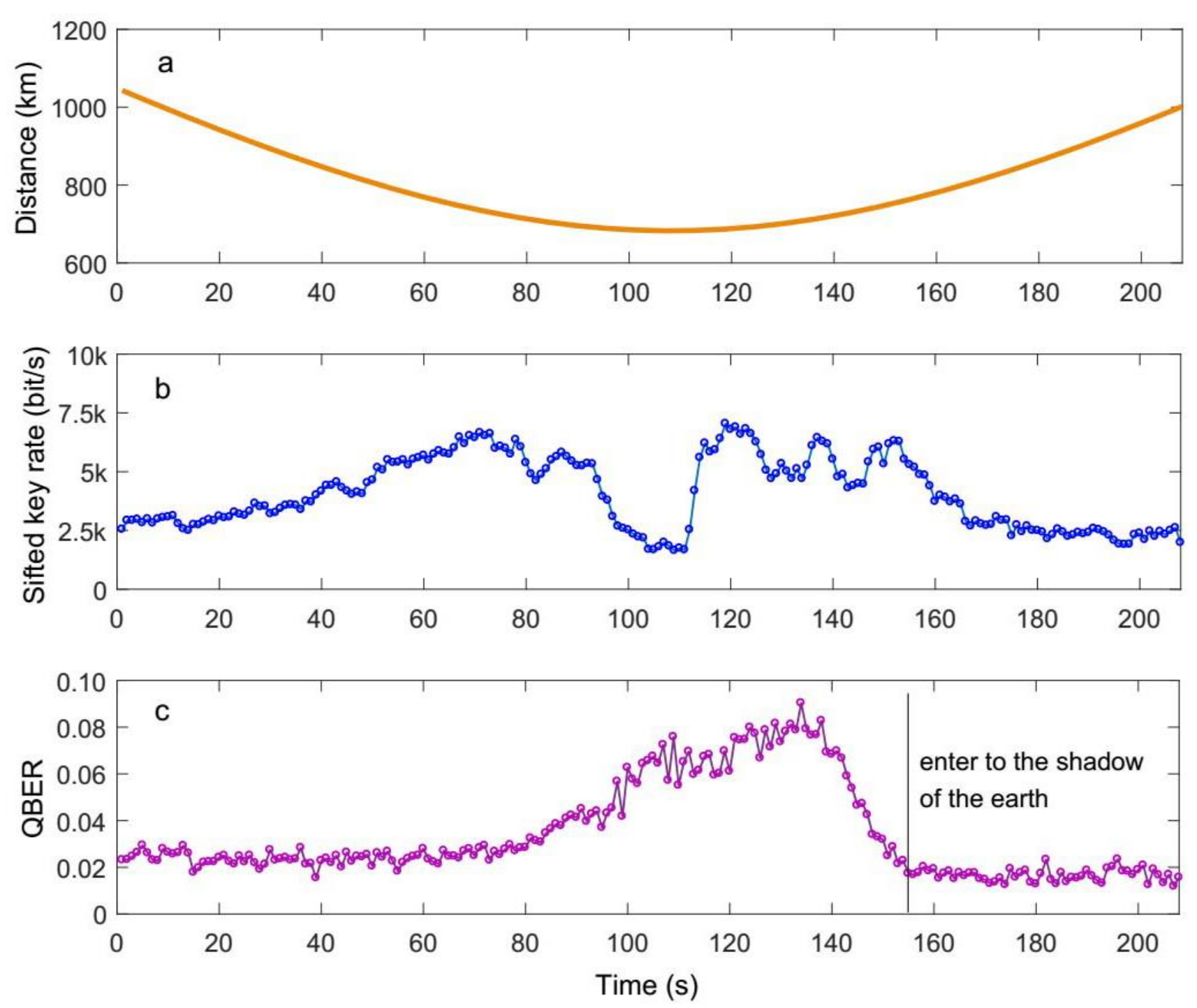


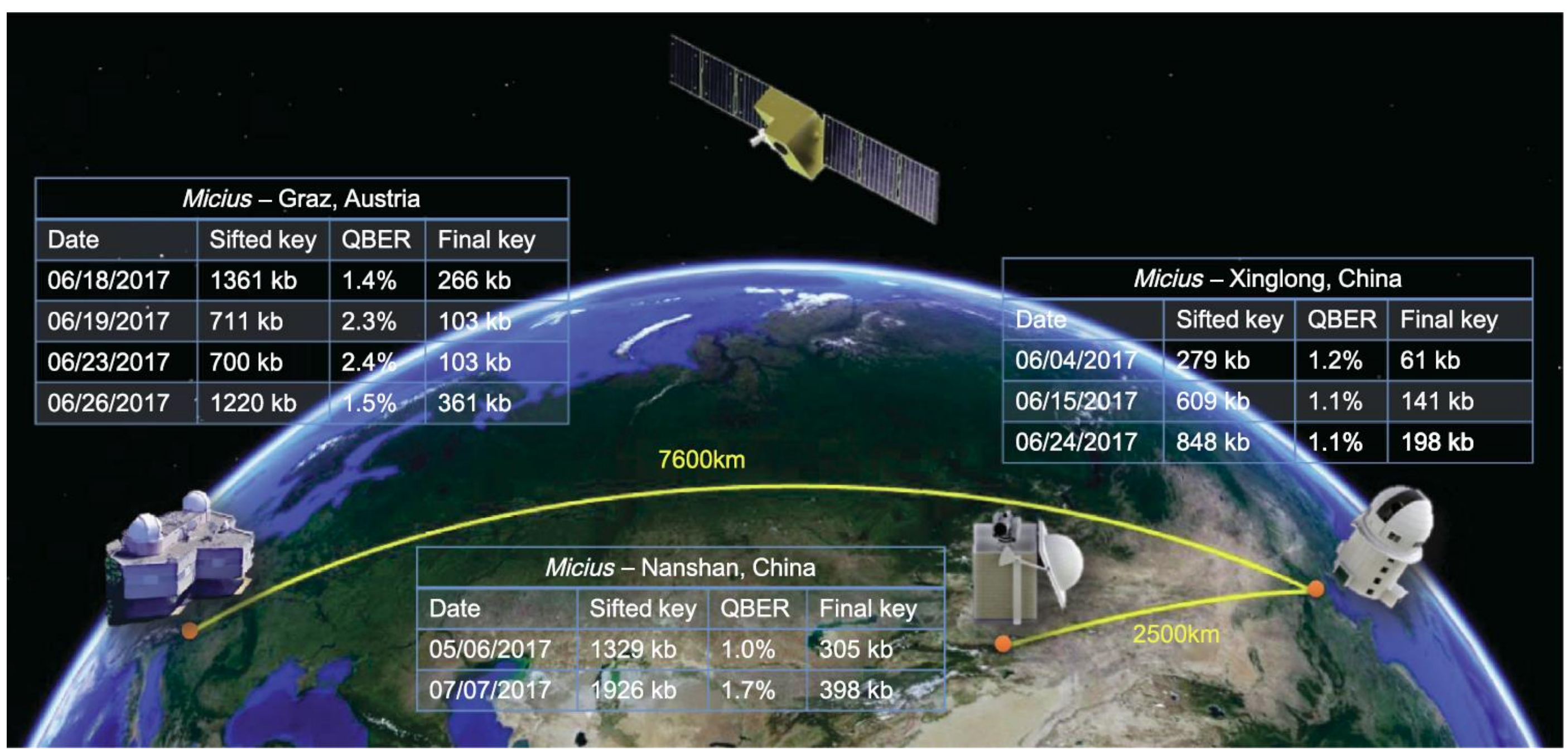




\section{One-Time-Pad Encryption}

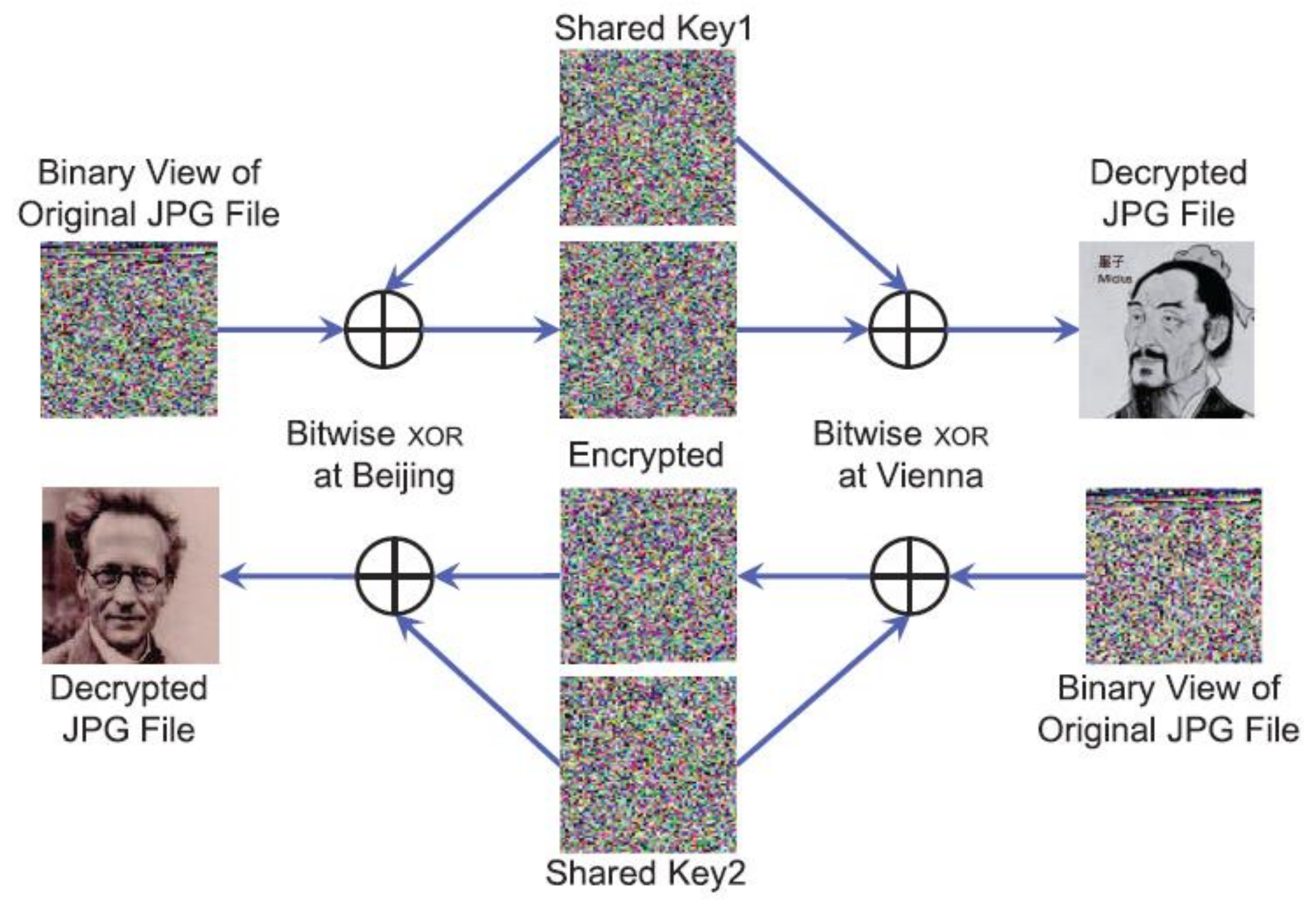




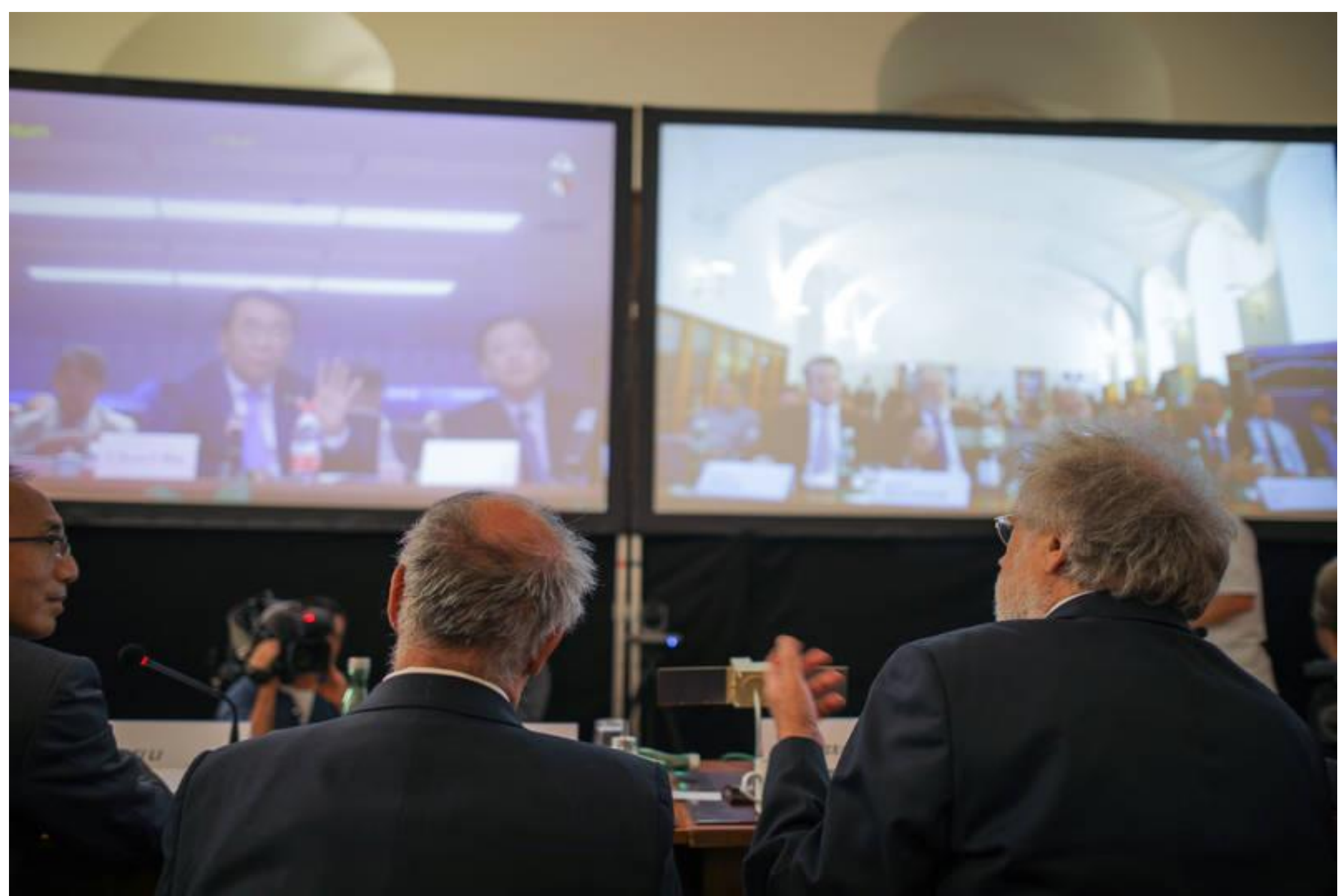

quantum keys used as 128bit AES seed and renewed every second 


\section{Team}
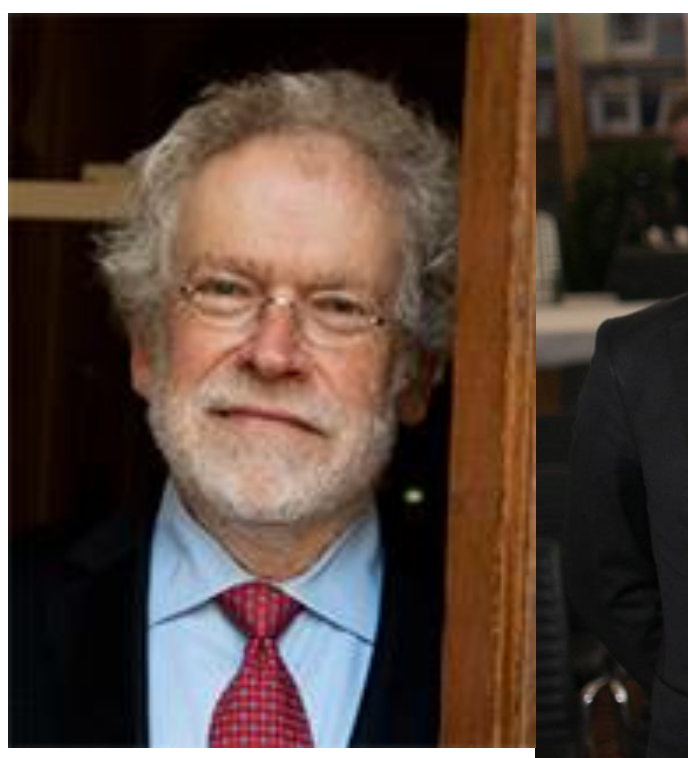

A. Zeilinger $+$

T. Herbst

D. Bricher

A. Rodriguez N. Fleischmann

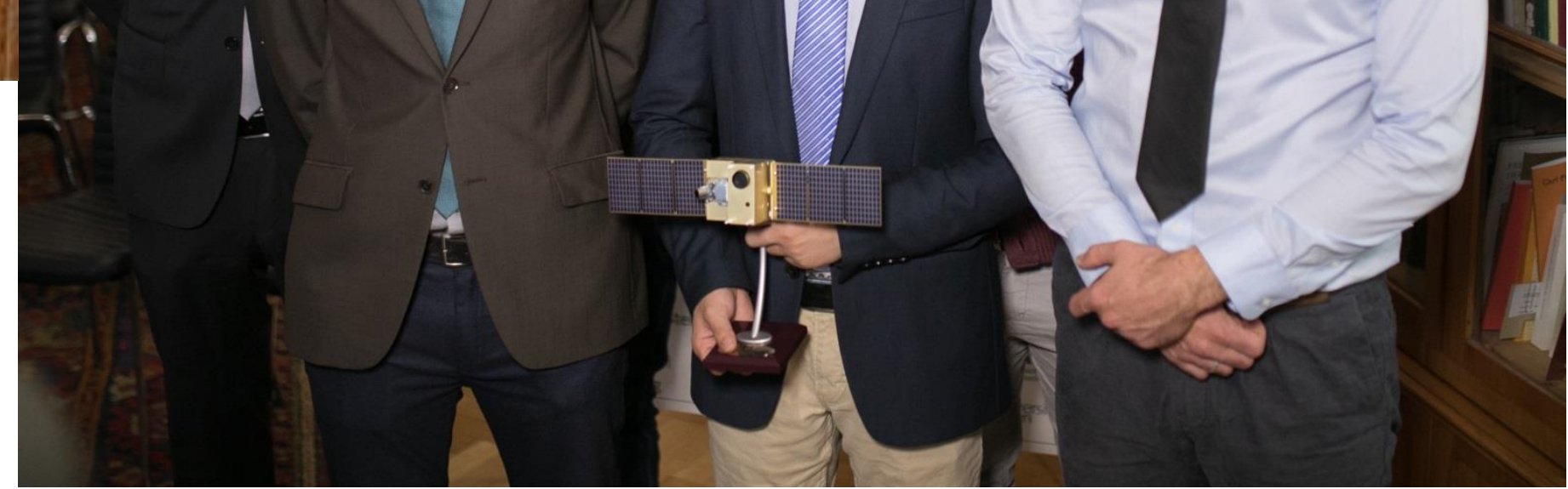

R. Ursin, J. Handsteiner, M. Fink, B. Liu, D. Rauch, T. Scheidl 
Thank you 


\section{Mobile Optical Ground Station}

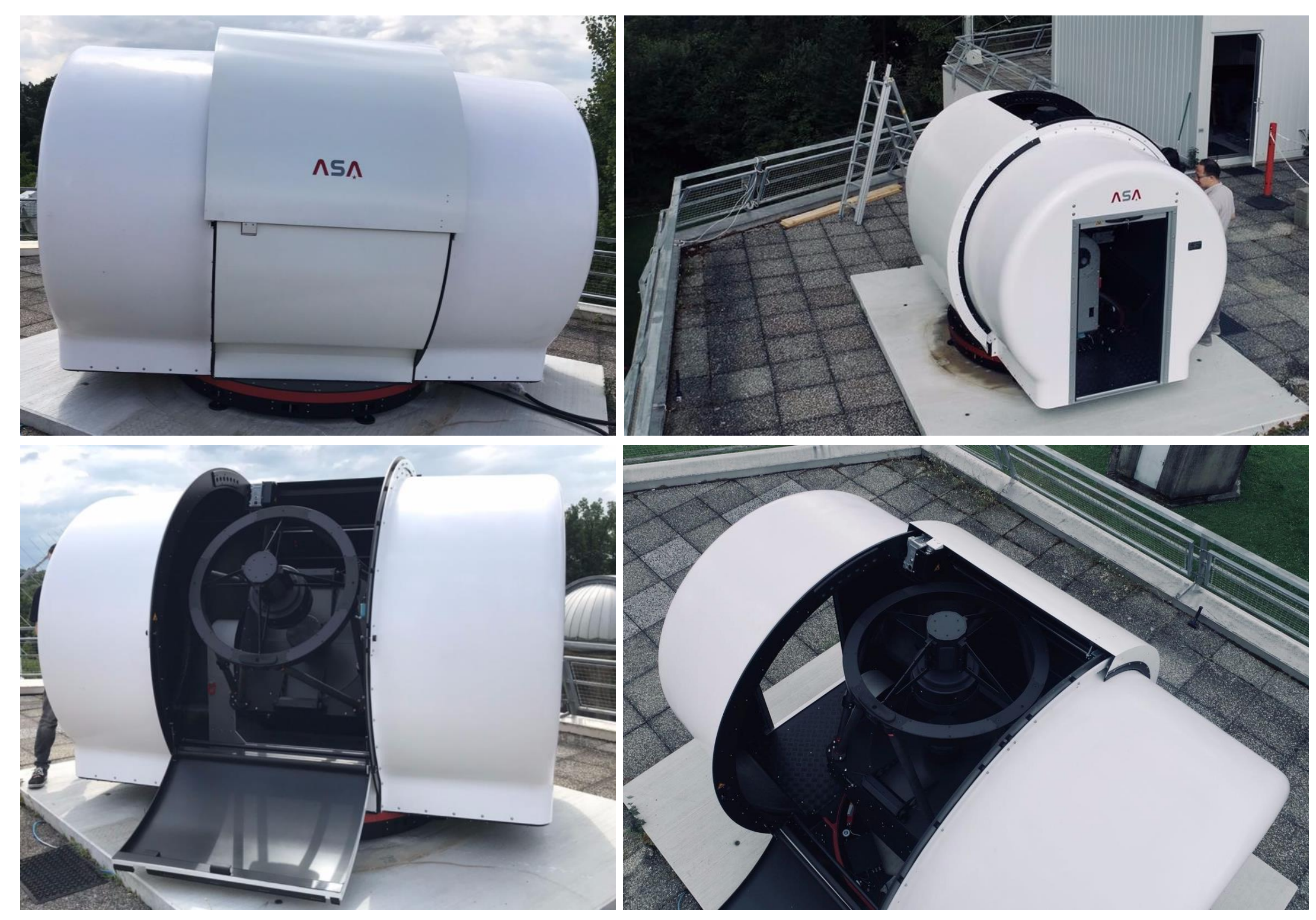

IQI

Vienna

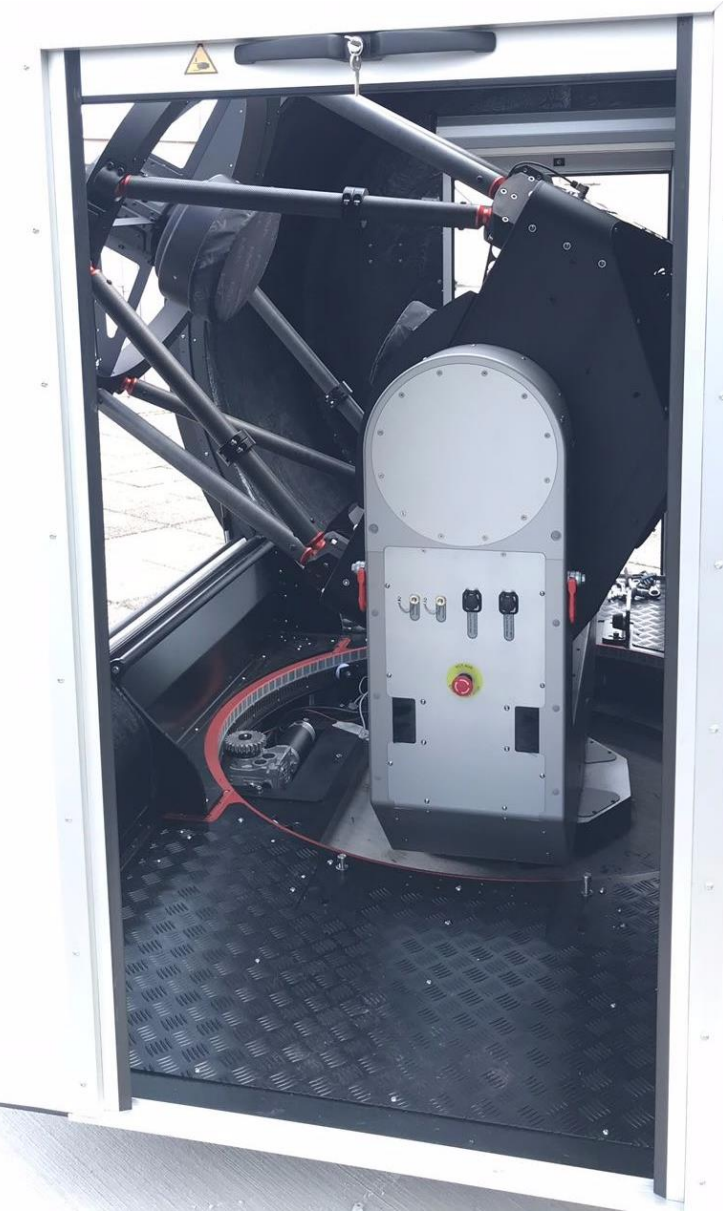

Optical System: $80 \mathrm{~cm}, \mathrm{f} / 6$ 


\section{Cryptography}

ÖSTERREICHISCHE AKADEMIE DER WISSENSCHAFTEN

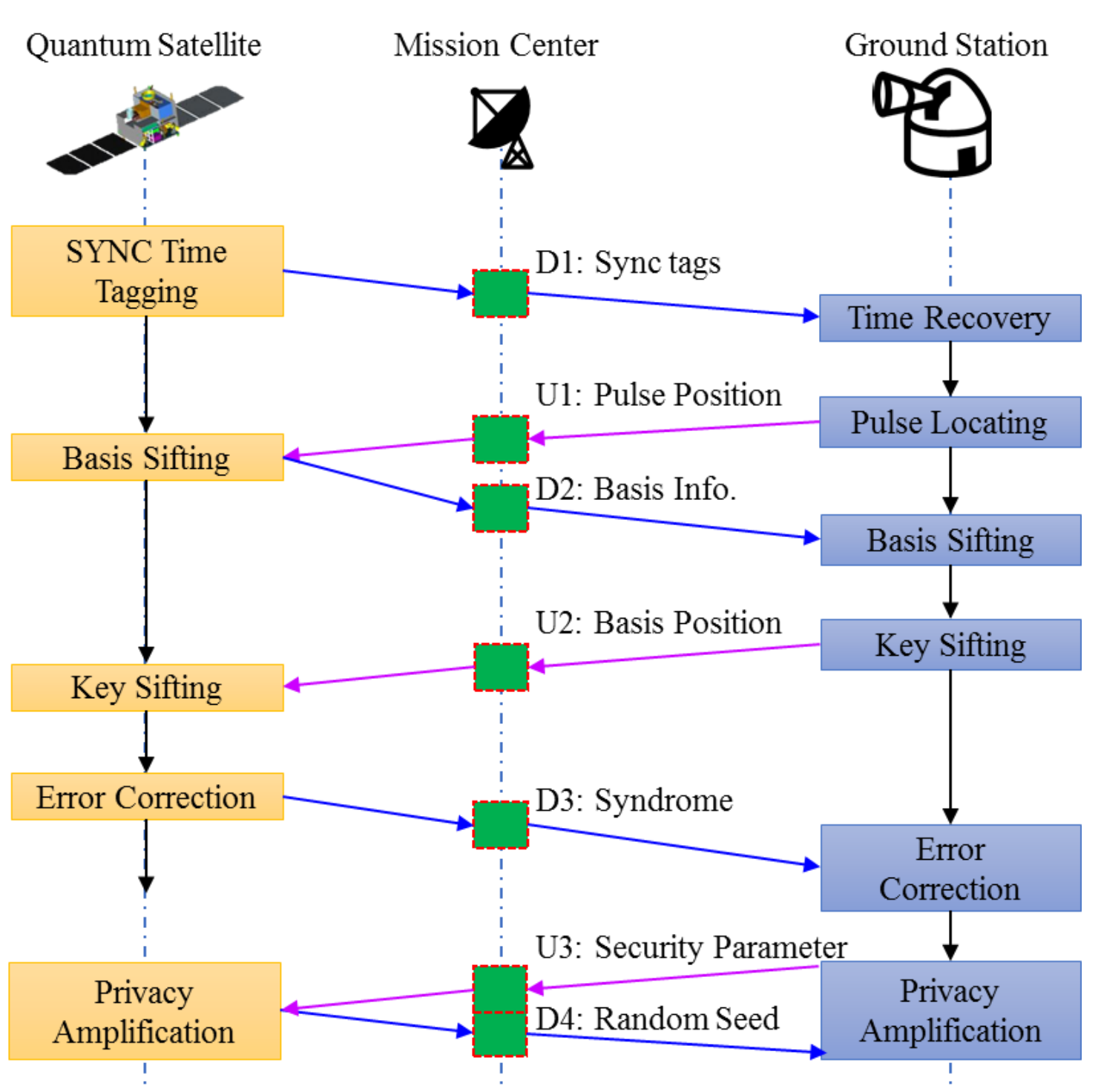




\section{Asymmetric cryptosystem:}

Alice gets an open padlock Bob only holds the key

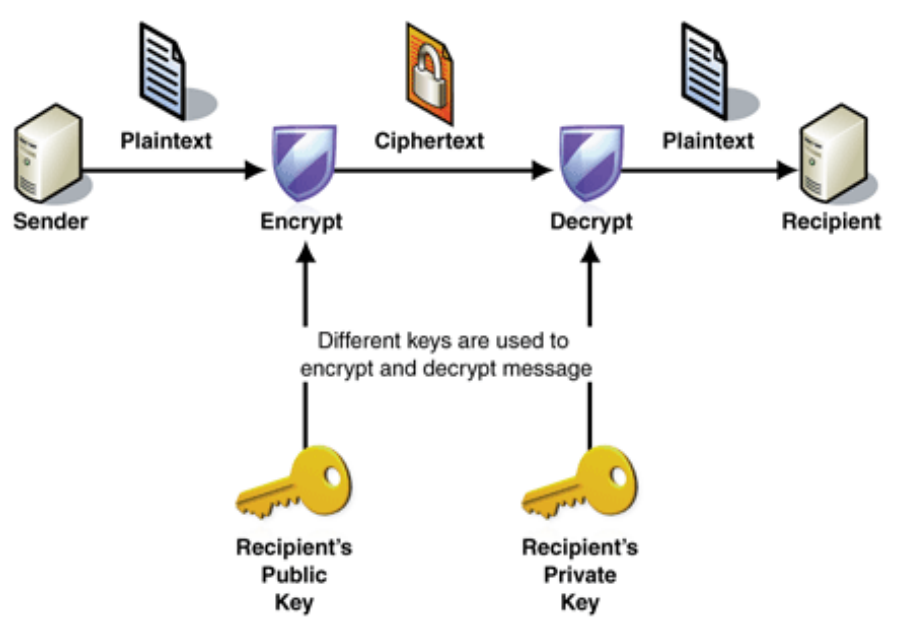

\section{Security based on computational} complexity, "RSA"-encryption

Rivest, R., A. Shamir, L. Adleman, ACM 21 (2), 120 -126 (1978)

no security proof
Symmetric cryptosystem is used as an algorithm:

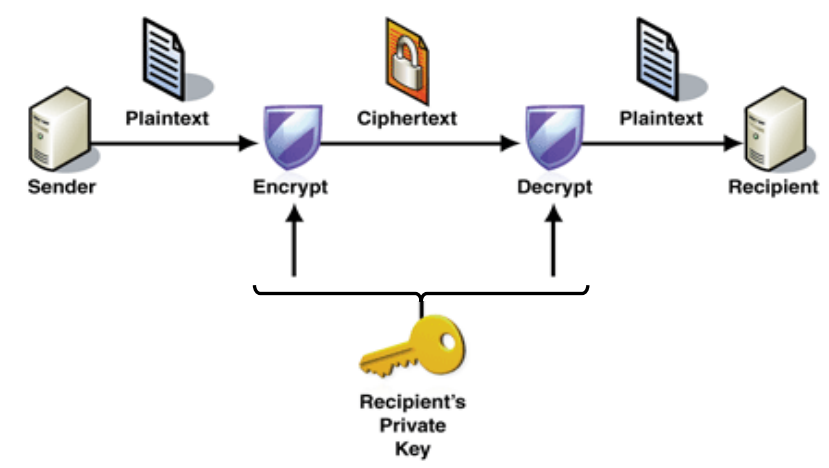

1. Encryption: Plaintext $(\mathrm{m}) \oplus$ Key $(\mathrm{k})=$ Ciphertext $(\mathrm{s})$

2. Transmission: Ciphertext is sent to Bob

3. Decryption: $s \ominus k=m \oplus k \ominus k=m$

\begin{tabular}{|c|c|c|c|c|c|c|c|c|c|c|c|c|c|c|c|c|}
\hline Cleartext $\mathrm{m}$ & 1 & 1 & 1 & 1 & 1 & 0 & 1 & 0 & 0 & 1 & 0 & 1 & 1 & 1 & 0 & 1 \\
\hline Key k & 1 & 0 & 0 & 1 & 0 & 0 & 1 & 1 & 1 & 1 & 0 & 0 & 1 & 0 & 1 & 1 \\
\hline Ciphertext s & 0 & 1 & 1 & 0 & 1 & 0 & 0 & 1 & 1 & 0 & 0 & 1 & 0 & 1 & 1 & 0 \\
\hline
\end{tabular}

Unconditionally secure if

- Key and message have the same length

- Key is used only once - "one-time- pad"

"One-time pad",

Vernam, G. S. J. Am. Inst.

Elec. Eng., 55, 10 (1926)

- Key is "truly" random

\section{Quantum Mechanics}

Heisenberg uncertainty relation

Non-Cloning Theorem 
No practical true single-photon sources yet available $\rightarrow$ faint laser pulses with $\mu<1$
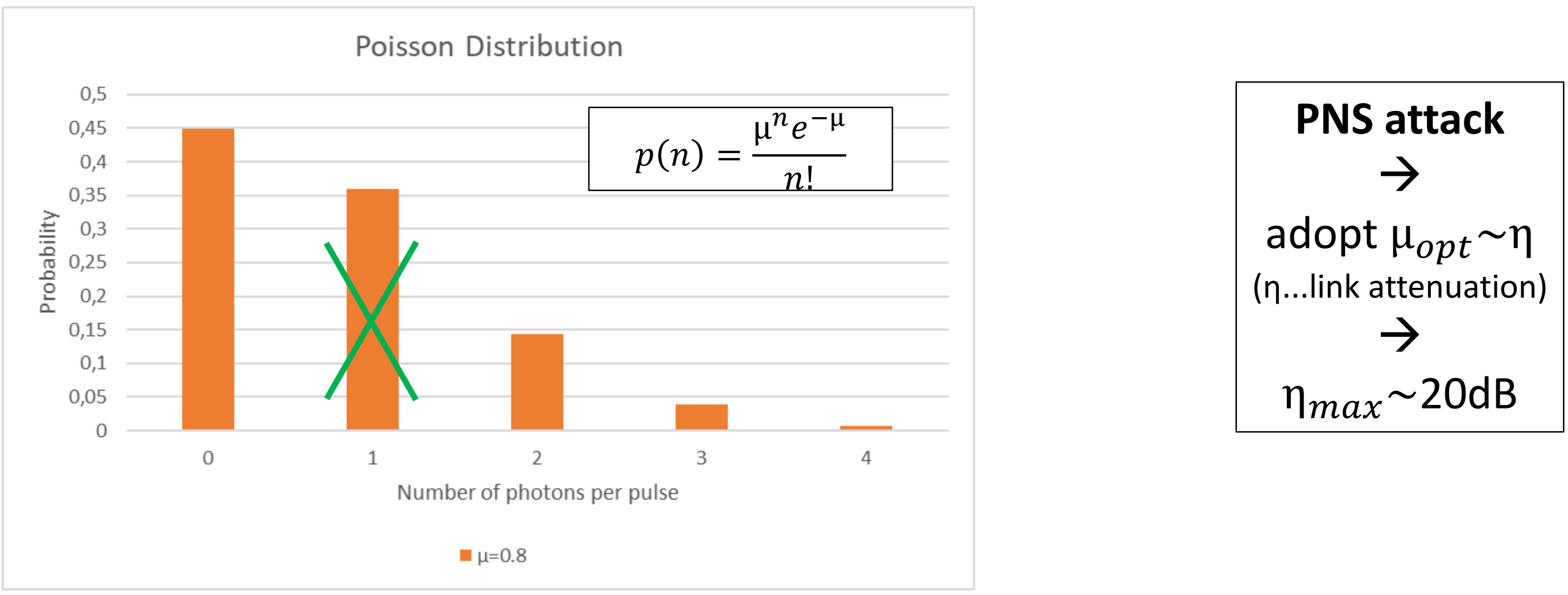
Quantum Science Satellite - MICIUS

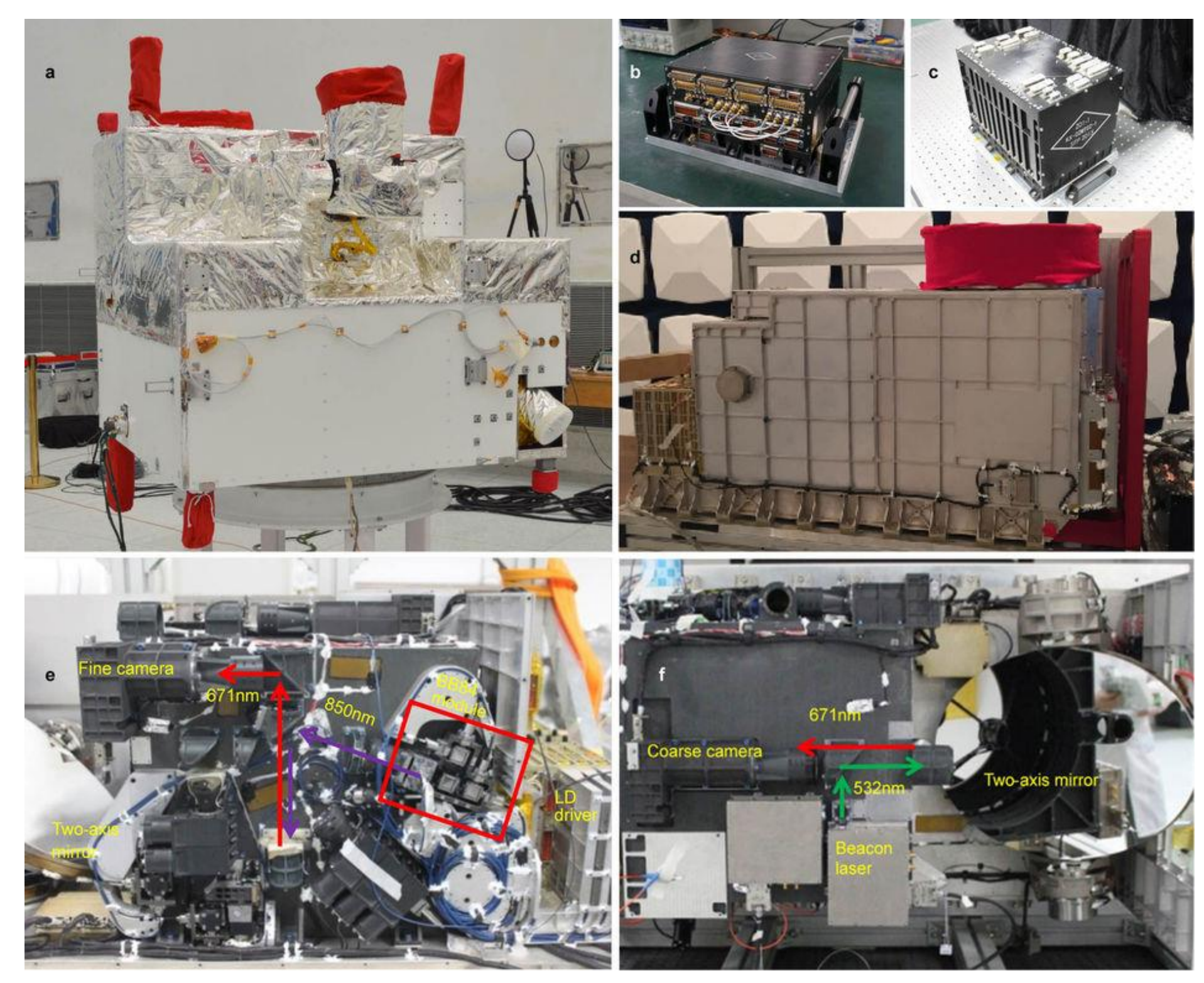

ÖSTERREICHISCHE

AKADEMIE DER

WISSENSCHAFTEN

I@I 


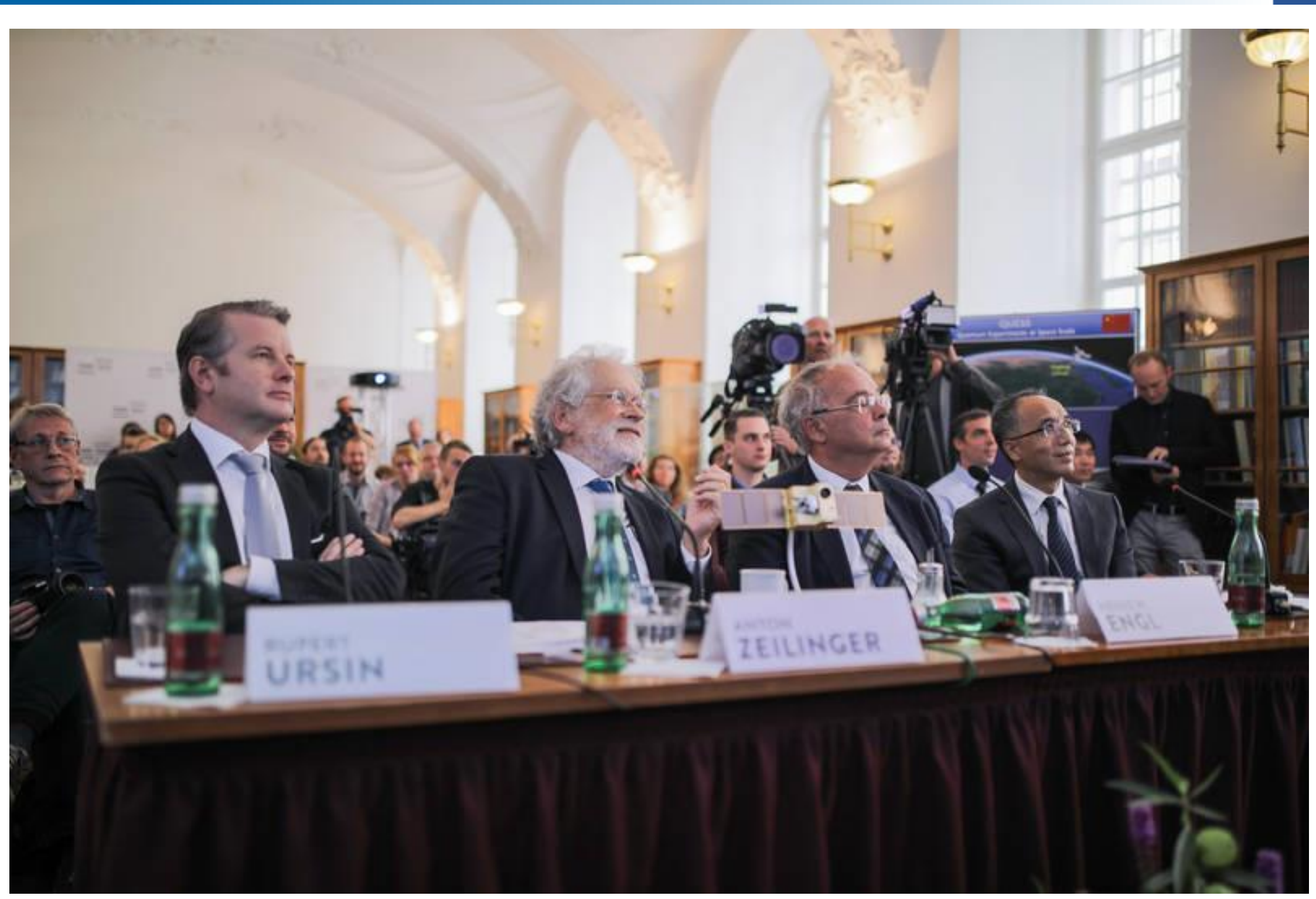



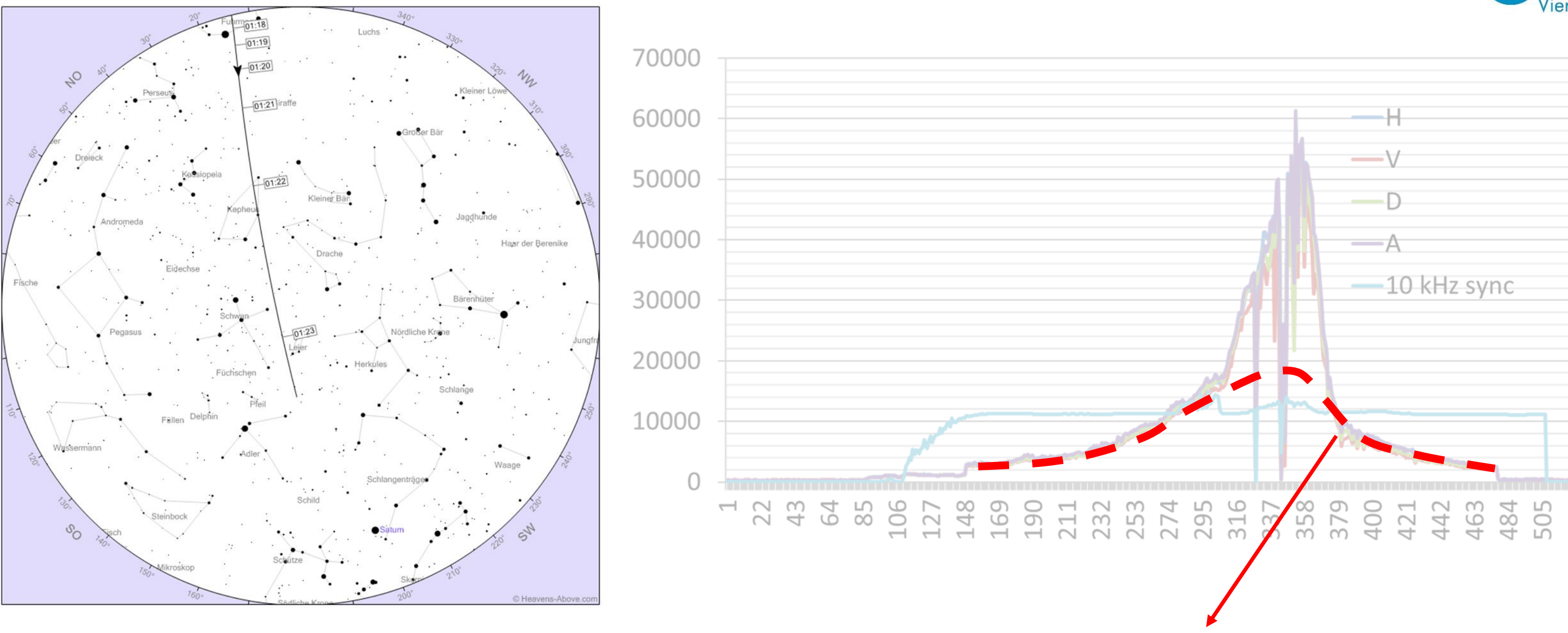


\section{SLR Station - Graz (Austria)}

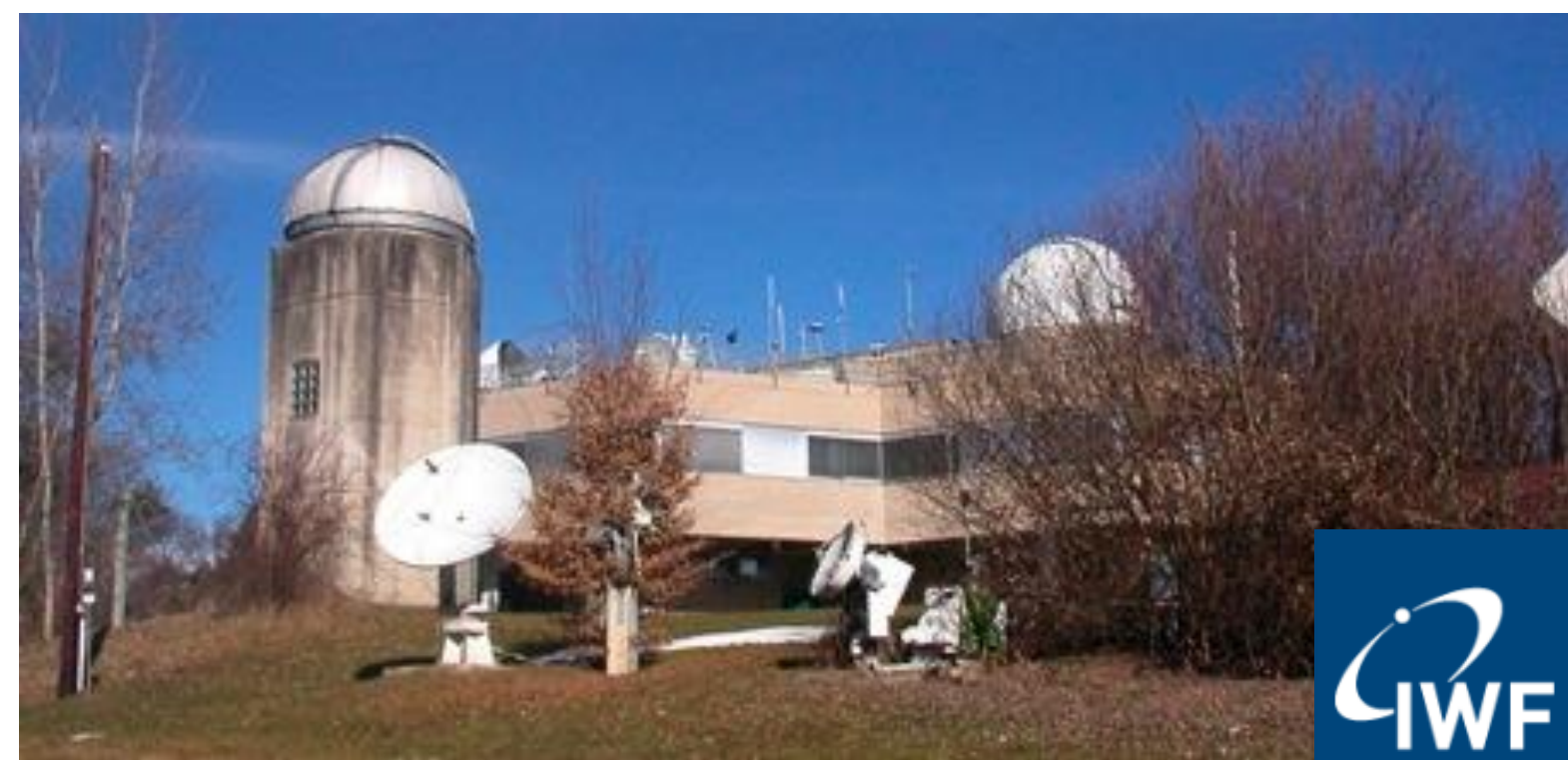

Satellite Laser Ranging Station - Graz Lustbühel

Aperture Size $-50 \mathrm{~cm}$ Location - 5km from Graz City Center

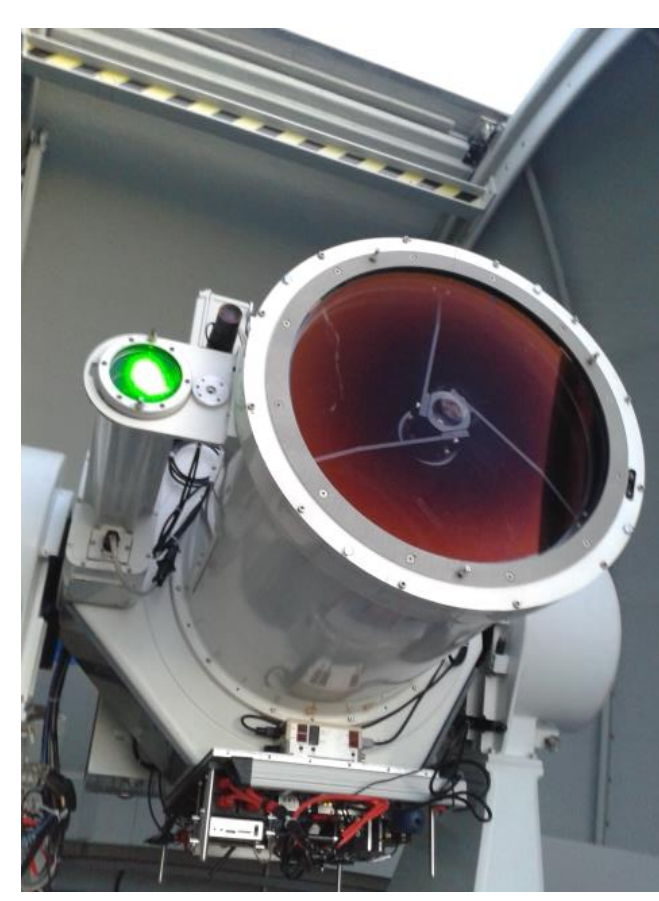




\section{Outline}

- Basics of Quantum Key Distribution

- Characteristics of „Micius“

- Optical Ground Station in Graz (Austria)

- OGS Performance Characteristics

- Tracking

- Polarization Compensation

- Results 